\title{
Small-x Evolution of Structure Functions in the Next-to-Leading Order ${ }^{1}$
}

\author{
Giovanni A. Chirilli \\ Physics Dept, Old Dominion Univ., Norfolk, VA 23529, and \\ Theory Group, JLab, 12000 Jefferson Ave, Newport News, VA 23606 \\ E-mail: chirilli@jlab.org
}

\begin{abstract}
The high-energy behavior of amplitudes in gauge theories can be reformulated in terms of the evolution of Wilson-line operators. In the leading order this evolution is governed by the nonlinear Balitsky-Kovchegov (BK) equation. The NLO corrections define the scale of the runningcoupling constant in the BK equation and in QCD, its kernel has both conformal and non-conformal parts. To separate the conformally invariant effects from the running-coupling effects, we calculate the NLO evolution of the color dipoles in the conformal $\mathscr{N}=4$ SYM theory, then we define the "composite dipole operator" with the rapidity cutoff preserving conformal invariance, and the resulting Möbius invariant kernel for this operator agrees with the forward NLO BFKL calculation.

In QCD, the NLO kernel for the composite operators resolves in a sum of the conformal part and the running-coupling part.
\end{abstract}

Keywords: Small- $x_{B}$ evolution, Wilson lines.

PACS: $12.38 . \mathrm{Bx}, 12.38 . \mathrm{Cy}$

\section{SMALL- $x_{B}$ EVOLUTION OF COLOR DIPOLES}

The high-energy scattering in a gauge theory can be described in terms of Wilson lines - infinite gauge factors ordered along the straight lines (see e.g. the review [2]). Indeed, the fast particle moves along its straight-line classical trajectory and the only quantum effect is the eikonal phase factor acquired along this propagation path. In QCD, for fast quark or gluon scattering off some target, this eikonal phase factor is a Wilson line - an infinite gauge link ordered along the straight line collinear to particle's velocity $n^{\mu}$ :

$$
U^{\eta}\left(x_{\perp}\right)=\operatorname{Pexp}\left\{i g \int_{-\infty}^{\infty} d u n_{\mu} A^{\mu}\left(u n+x_{\perp}\right)\right\}
$$

Here $A_{\mu}$ is the gluon field of the target, $x_{\perp}$ is the transverse position of the particle which remains unchanged throughout the collision, and the index $\eta$ labels the rapidity of the particle.

The high-energy behavior of QCD amplitudes can be studied in the framework of the evolution of color dipoles. Let us consider the small- $x$ behavior of structure functions of deep inelastic scattering (DIS). At high energies the virtual photon decomposes into quark and antiquark which propagate along the straight lines separated by transverse

\footnotetext{
1 Talk given at CIPANP 2009: Tenth Conference on the Intersections of Particle and Nuclear Physics, Torrey Pines Hilton, San Diego, California, 26 May to 31 May, 2009, based on [1].
} 
distance and form a color dipole - two-Wilson-line operator.

$$
\hat{\mathscr{U}}^{\eta}\left(x_{\perp}, y_{\perp}\right)=1-\frac{1}{N_{c}} \operatorname{tr}\left\{\hat{U}^{\eta}\left(x_{\perp}\right) \hat{U}^{\dagger \eta}\left(y_{\perp}\right)\right\}
$$

The energy dependence of the structure function is then translated into the dependence of the color dipole on the rapidity $\eta$. There are two ways to restrict the rapidity of Wilson lines: one can consider Wilson lines with the support line collinear to the velocity of the fast-moving particle or one can take the light-like Wilson line and cut the rapidity integrals "by hand". While the former method appears to be more natural, it is technically simpler to get the conformal results with the latter method of "rigid cutoff" in the longitudinal direction.

Thus, the small-x behavior of the structure functions is governed by the rapidity evolution of color dipoles $[3,4]$. At relatively high energies and for sufficiently small dipoles we can use the leading logarithmic approximation (LLA) where $\alpha_{S} \ll 1, \alpha_{S} \ln x_{B} \sim 1$ and get the non-linear BK evolution equation for the color dipoles [5, 6]:

$$
\begin{aligned}
\frac{d}{d \eta} \hat{\mathscr{U}}^{\eta}\left(z_{1}, z_{2}\right)= & \frac{\alpha_{S} N_{c}}{2 \pi^{2}} \int d^{2} z_{3} \\
& \frac{z_{12}^{2}}{z_{13}^{2} z_{23}^{2}}\left[\hat{\mathscr{U}}^{\eta}\left(z_{1}, z_{3}\right)+\hat{\mathscr{U}}^{\eta}\left(z_{3}, z_{2}\right)\right) \\
& \left.-\hat{\mathscr{U}}^{\eta}\left(z_{1}, z_{3}\right)-\hat{\mathscr{U}}^{\eta}\left(z_{1}, z_{3}\right) \hat{\mathscr{U}}^{\eta}\left(z_{3}, z_{2}\right)\right]
\end{aligned}
$$

where $\eta=\ln \frac{1}{x_{B}}$ and $z_{12} \equiv z_{1}-z_{2}$ etc. (As usual, we denote operators by "hat"). The first three terms correspond to the linear BFKL evolution [7] and describe the parton emission while the last term is responsible for the parton annihilation. For sufficiently low $x_{B}$ the parton emission balances the parton annihilation so the partons reach the state of saturation [8] with the characteristic transverse momentum $Q_{s}$ growing with energy $1 / x_{B}$ (for a review, see [9])

It is easy to see that the BK equation (3) is conformally invariant in the twodimensional space. This follows from the conformal invariance of the light-like Wilson lines. Indeed, the Wilson line

$$
U\left(x_{\perp}\right)=\operatorname{Pexp}\left\{i g \int_{-\infty}^{\infty} d x^{+} A_{+}\left(x^{+}, x_{\perp}\right)\right\}
$$

is invariant under the inversion $x^{\mu} \rightarrow x^{\mu} / x^{2}$ (with respect to the point with zero (-) component). Indeed, $\left(x^{+}, x_{\perp}\right)^{2}=-x_{\perp}^{2}$ so after the inversion $x_{\perp} \rightarrow x_{\perp} / x_{\perp}^{2}$ and $x^{+} \rightarrow$ $x^{+} / x_{\perp}^{2}$ and therefore

$$
U\left(x_{\perp}\right) \rightarrow \operatorname{Pexp}\left\{i g \int_{-\infty}^{\infty} d \frac{x^{+}}{x_{\perp}^{2}} A_{+}\left(\frac{x^{+}}{x_{\perp}^{2}}, x_{\perp}\right)\right\}=U\left(x_{\perp} / x_{\perp}^{2}\right)
$$

It is easy to check that the Wilson line operators lie in the standard representation of the conformal Möbius group SL(2,C) with conformal spin 0.

\section{NLO EVOLUTION OF COLOR DIPOLES}

The NLO evolution of color dipole in QCD [10] is not expected to be Möbius invariant due to the conformal anomaly leading to dimensional transmutation and running cou- 
pling constant. However, the NLO BK equation in QCD [10] has an additional term violating Möbius invariance and not related to the conformal anomaly. To understand the relation between the high-energy behavior of amplitudes and Möbius invariance of Wilson lines, it is instructive to consider the conformally invariant $\mathscr{N}=4$ super YangMils theory. This theory was intensively studied in recent years due to the fact that at large coupling constants it is dual to the IIB string theory in the $\mathrm{AdS}_{5}$ background. In the light-cone limit, the contribution of scalar operators to Maldacena-Wilson line [11] vanishes so one has the usual Wilson line constructed from gauge fields and therefore the LLA evolution equation for color dipoles in the $\mathscr{N}=4 \mathrm{SYM}$ has the same form as (3). At the NLO level, the contributions from gluino and scalar loops enter the picture.

As we mentioned above, formally the light-like Wilson lines are Möbius invariant. Unfortunately, the light-like Wilson lines are divergent in the longitudinal direction and moreover, it is exactly the evolution equation with respect to this longitudinal cutoff which governs the high-energy behavior of amplitudes. At present, it is not known how to find the conformally invariant cutoff in the longitudinal direction. When we use the non-invariant cutoff we expect, as usual, the invariance to hold in the leading order but to be violated in higher orders in perturbation theory. In our calculation we restrict the longitudinal momentum of the gluons composing Wilson lines, and with this noninvariant cutoff the NLO evolution equation in QCD has extra non-conformal parts not related to the running of coupling constant. Similarly, there will be non-conformal parts coming from the longitudinal cutoff of Wilson lines in the $\mathscr{N}=4 \mathrm{SYM}$ equation. In [1] we demonstrate that it is possible to construct the "composite conformal dipole operator" (order by order in perturbation theory) which mimics the conformal cutoff in the longitudinal direction so the corresponding evolution equation has no extra nonconformal parts. This is similar to the construction of the composite renormalized local operator in the case when the UV cutoff does not respect the symmetries of the bare operator - in this case the symmetry of the UV-regularized operator is preserved order by order in perturbation theory by subtraction of the symmetry-restoring counterterms.

Let us present our result for the NLO evolution of the color dipole in the adjoint representation (hereafter we use notations $z_{i j} \equiv z_{i}-z_{j}$ and $\left(T^{a}\right)_{b c}=-i f^{a b c}$ )

$$
\begin{aligned}
& \frac{d}{d \eta}\left[\operatorname{Tr}\left\{\hat{U}_{z_{1}}^{\eta} \hat{U}_{z_{2}}^{\dagger \eta}\right\}\right]^{\operatorname{conf}} \\
& =\frac{\alpha_{s}}{\pi^{2}} \int d^{2} z_{3} \frac{z_{12}^{2}}{z_{13}^{2} z_{23}^{2}}\left[1-\frac{\alpha_{s} N_{c}}{4 \pi} \frac{\pi^{2}}{3}\right]\left[\operatorname{Tr}\left\{T^{a} \hat{U}_{z_{1}}^{\eta} \hat{U}_{z_{3}}^{\dagger \eta} T^{a} \hat{U}_{z_{3}} \hat{U}_{z_{2}}^{\dagger \eta}\right\}-N_{c} \operatorname{Tr}\left\{\hat{U}_{z_{1}}^{\eta} \hat{U}_{z_{2}}^{\dagger \eta}\right\}\right]^{\mathrm{conf}} \\
& \quad-\frac{\alpha_{s}^{2}}{4 \pi^{4}} \int d^{2} z_{3} d^{2} z_{4} \frac{z_{12}^{2}}{z_{13}^{2} z_{24}^{2} z_{34}^{2}}\left\{2 \ln \frac{z_{12}^{2} z_{34}^{2}}{z_{14}^{2} z_{23}^{2}}+\left[1+\frac{z_{12}^{2} z_{34}^{2}}{z_{13}^{2} z_{24}^{2}-z_{14}^{2} z_{23}^{2}}\right] \ln \frac{z_{13}^{2} z_{24}^{2}}{z_{14}^{2} z_{23}^{2}}\right\} \\
& \left.\quad \times \operatorname{Tr}\left\{\left[T^{a}, T^{b}\right] \hat{U}_{z_{1}}^{\eta} T^{a^{\prime}} T^{b^{\prime}} \hat{U}_{z_{2}}^{\dagger \eta}+T^{b} T^{a} \hat{U}_{z_{1}}^{\eta}\left[T^{b^{\prime}}, T^{a^{\prime}}\right] \hat{U}_{z_{2}}^{\dagger \eta}\right\}\left[\left(\hat{U}_{z_{3}}^{\eta}\right)\right)^{a a^{\prime}}\left(\hat{U}_{z_{4}}^{\eta}\right)^{b b^{\prime}}-\left(z_{4} \rightarrow z_{3}\right)\right]
\end{aligned}
$$

where

$$
\begin{array}{r}
{\left[\operatorname{Tr}\left\{\hat{U}_{z_{1}}^{\eta} \hat{U}_{z_{2}}^{\dagger \eta}\right\}\right]^{\mathrm{conf}}=\operatorname{Tr}\left\{\hat{U}_{z_{1}}^{\eta} \hat{U}_{z_{2}}^{\dagger \eta}\right\}+\frac{\alpha_{s}}{2 \pi^{2}} \int d^{2} z_{3} \frac{z_{12}^{2}}{z_{13}^{2} z_{23}^{2}}\left[\operatorname{Tr}\left\{T^{n} \hat{U}_{z_{1}}^{\eta} \hat{U}_{z_{3}}^{\dagger \eta} T^{n} \hat{U}_{z_{3}}^{\eta} \hat{U}_{z_{2}}^{\dagger \eta}\right\}\right.} \\
\left.-N_{c} \operatorname{Tr}\left\{\hat{U}_{z_{1}}^{\eta} \hat{U}_{z_{2}}^{\dagger \eta}\right\}\right] \ln \frac{a z_{12}^{2}}{z_{13}^{2} z_{23}^{2}}
\end{array}
$$


is the "composite dipole" with the conformal longitudinal cutoff in the next-to-leading order and $a$ is an arbitrary dimensional constant. In fact, $a(\eta)=a e^{\eta}$ plays the same role for the rapidity evolution as $\mu^{2}$ for the usual DGLAP evolution: the derivative $\frac{d}{d a}$ gives the evolution equation (6). The kernel in the r.h.s. of Eq. (6) is obviously Möbius invariant since it depends on two four-point conformal ratios $\frac{z_{13}^{2} z_{24}^{2}}{z_{14}^{2} z_{23}^{2}}$ and $\frac{z_{12}^{2} z_{34}^{2}}{z_{13}^{2} z_{24}^{2}}$. In [1] we also demonstrate that Eq. (6) agrees with forward NLO BFKL calculation of Ref. [12].

\section{ACKNOWLEDGMENTS}

This work was supported by DOE grant DE-FG02-97ER41028 and by the Jefferson Lab Graduate Fellowship. The author thanks the organizers for the warm hospitality received during this conference.

\section{REFERENCES}

1. I. Balitsky and G.A. Chirilli, arXiv:0903.5326 [hep-ph].

2. I. Balitsky, "High-Energy QCD and Wilson Lines", In *Shifman, M. (ed.): At the frontier of particle physics, vol. 2*, p. 1237-1342 (World Scientific, Singapore,2001) [hep-ph/0101042]

3. A.H. Mueller, Nucl. Phys. B415, 373 (1994); A.H. Mueller and Bimal Patel, Nucl. Phys. B425, 471 (1994).

4. N.N. Nikolaev and B.G. Zakharov, Phys. Lett. B 332, 184 (1994); Z. Phys. C64, 631 (1994); N.N. Nikolaev B.G. Zakharov, and V.R. Zoller, JETP Letters 59, 6 (1994).

5. I. Balitsky, Nucl. Phys. B463, 99 (1996); “Operator expansion for diffractive high-energy scattering”, [hep-ph/9706411];

6. Yu.V. Kovchegov, Phys. Rev. D60, 034008 (1999); Phys. Rev. D61,074018 (2000).

7. V.S. Fadin, E.A. Kuraev, and L.N. Lipatov, Phys. Lett. B 60, 50 (1975); I. Balitsky and L.N. Lipatov, Sov. Journ. Nucl. Phys. 28, 822 (1978).

8. L.V. Gribov, E.M. Levin, and M.G. Ryskin, Phys. Rept. 100, 1 (1983), A.H. Mueller and J.W. Qiu, Nucl. Phys. B268, 427 (1986); A.H. Mueller, Nucl. Phys. B335, 115 (1990).

9. E. Iancu and R. Venugopalan , In *Hwa, R.C. (ed.) et al.: Quark gluon plasma* 249-3363, [e-Print: hep-ph/0303204];

H. Weigert, Prog.Part.Nucl.Phys.55, 461(2005);

J. Jalilian-Marian and Yu.V. Kovchegov, Prog.Part.Nucl.Phys.56, 104(2006).

10. I. Balitsky and G.A. Chirilli, Phys.Rev.D77, 014019(2008)

11. J.M. Maldacena, Phys.Rev.Lett. 80, 4859 (1998).

12. A.V. Kotikov and L.N. Lipatov, Nucl. Phys. B582, 19 (2000); Nucl. Phys. B5661, 19 (2003). Erratumibid., B685, 405 (2004). 